\title{
Hints of Spin-Orbit Resonances in the Binary Black Hole Population
}

\author{
Vijay Varma $\odot,{ }^{1,2,3, *}$ Sylvia Biscoveanu $\odot,{ }^{4,5}$ Maximiliano Isi $\odot,{ }^{4,5}$ Will M. Farr, ${ }^{6,7}$ and Salvatore Vitale ${ }^{4,5}$ \\ ${ }^{1}$ Department of Physics, Cornell University, Ithaca, New York 14853, USA \\ ${ }^{2}$ Cornell Center for Astrophysics and Planetary Science, Cornell University, Ithaca, New York 14853, USA \\ ${ }^{3}$ Max Planck Institute for Gravitational Physics (Albert Einstein Institute), Am Mühlenberg 1, Potsdam 14476, Germany \\ ${ }^{4}$ LIGO Laboratory, Massachusetts Institute of Technology, Cambridge, Massachusetts 02139, USA \\ ${ }^{5}$ Department of Physics and Kavli Institute for Astrophysics and Space Research, Massachusetts Institute of Technology, \\ 77 Massachusetts Avenue, Cambridge, Massachusetts 02139, USA \\ ${ }^{6}$ Department of Physics and Astronomy, Stony Brook University, Stony Brook, New York 11794, USA \\ ${ }^{7}$ Center for Computational Astrophysics, Flatiron Institute, New York, New York 10010, USA
}

(Received 23 July 2021; revised 29 September 2021; accepted 17 December 2021; published 19 January 2022)

Binary black hole spin measurements from gravitational wave observations can reveal the binary's evolutionary history. In particular, the spin orientations of the component black holes within the orbital plane, $\phi_{1}$ and $\phi_{2}$, can be used to identify binaries caught in the so-called spin-orbit resonances. In a companion paper, we demonstrate that $\phi_{1}$ and $\phi_{2}$ are best measured near the merger of the two black holes. In this work, we use these spin measurements to provide the first constraints on the full six-dimensional spin distribution of merging binary black holes. In particular, we find that there is a preference for $\Delta \phi=$ $\phi_{1}-\phi_{2} \sim \pm \pi$ in the population, which can be a signature of spin-orbit resonances. We also find a preference for $\phi_{1} \sim-\pi / 4$ with respect to the line of separation near merger, which has not been predicted for any astrophysical formation channel. However, the strength of these preferences depends on our prior choices, and we are unable to constrain the widths of the $\phi_{1}$ and $\Delta \phi$ distributions. Therefore, more observations are necessary to confirm the features we find. Finally, we derive constraints on the distribution of recoil kicks in the population and use this to estimate the fraction of merger remnants retained by globular and nuclear star clusters. We make our spin and kick population constraints publicly available.

DOI: 10.1103/PhysRevLett.128.031101

Introduction.-Binaries of spinning black holes (BHs) serve as a unique astrophysical laboratory for a range of relativistic phenomena. For example, if the $\mathrm{BH}$ spins $\chi_{1}$ and $\chi_{2}$ are aligned with the orbital angular momentum $\boldsymbol{L}$, the orientations of the orbital plane and the spins remain fixed during the inspiral (cf. Fig. 1 for definitions of the binary $\mathrm{BH}$ spin parameters). However, if the spins are tilted with respect to $\boldsymbol{L}$, relativistic spin-orbit and spinspin coupling cause the orbital plane and the spins to precess $[1,2]$.

While the tilt angles $\theta_{1}$ and $\theta_{2}$ control precession, the orbital-plane spin angles $\phi_{1}$ and $\phi_{2}$ play a central role in binaries undergoing spin-orbit resonances (SORs) [6]. For these binaries, the $\chi_{1}, \chi_{2}$, and $\boldsymbol{L}$ vectors become locked into a common resonant plane such that $\Delta \phi=\phi_{1}-\phi_{2}$ is fixed at 0 or $\pm \pi$ as the binary precesses. References $[7,8]$ pointed out that this locking is a limiting case of librating states near $\Delta \phi \sim 0$ or $\pm \pi$. For simplicity, we will follow previous

Published by the American Physical Society under the terms of the Creative Commons Attribution 4.0 International license. Further distribution of this work must maintain attribution to the author(s) and the published article's title, journal citation, and DOI. Open access publication funded by the Max Planck Society. literature [9-11] and refer to the more general librating states as SORs. While evidence for precession has been found in the astrophysical binary BH population [12], SORs have not yet been observed even though they are expected in some astrophysical scenarios. For example,

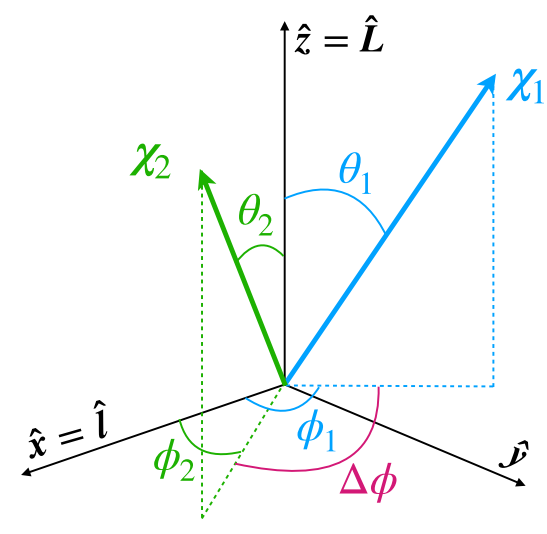

FIG. 1. The BH spins are represented by 3 -vectors $\chi_{1}$ and $\chi_{2}$, with index 1 (2) denoting the heavier (lighter) BH. We parameterize the spins by their dimensionless magnitudes $\chi_{1}, \chi_{2} \leq 1$, tilts $\theta_{1}, \theta_{2}$ with respect to the orbital angular momentum $\boldsymbol{L}$ [3], and orbital-plane spin angles $\phi_{1}, \phi_{2}$ with respect to the line of separation $\boldsymbol{l}$ from the lighter to the heavier BH. Finally, $\Delta \phi=\phi_{1}-\phi_{2}$. 
stellar binaries can cluster near these resonances if supernova natal kicks and stellar tides are significant $[13,14]$.

Another important relativistic effect that gets amplified for spinning binaries is the gravitational recoil. Gravitational waves (GWs) can carry away linear momentum from the binary, imparting a recoil or kick velocity to the merger remnant [15-18]. These velocities can reach values up to $\sim 5000 \mathrm{~km} / \mathrm{s}$ for precessing binaries [19-21], large enough to be ejected from any host galaxy [22]. Kick measurements from GW observations [23] can be used to constrain the formation of heavy $\mathrm{BHs}$ via successive mergers [24]. However, the kick depends very sensitively on the orbital-plane spin angles [25].

GW observations by LIGO [26] and Virgo [27] have enabled increasingly precise constraints on the astrophysical distributions of BH spin magnitudes and tilts $[12,28]$, but the distributions of the orbital-plane spin angles remain unconstrained. Constraining these distributions would allow us to understand the prevalence of SORs and merger kicks in nature. The biggest obstacle for this, however, is the difficulty in measuring $\phi_{1}, \phi_{2}$, and $\Delta \phi$ from individual GW events with current detectors [9-11,29-31].

However, in a companion paper, Varma et al. [32], we show that this can be greatly improved by measuring the spins near the merger, in particular, at a fixed "dimensionless" reference time $t_{\text {ref }} / M=-100$ before the peak of the GW amplitude, rather than the traditional choice of a fixed GW frequency of $f_{\text {ref }}=20 \mathrm{~Hz}$. Here $M=m_{1}+m_{2}$ is the total (redshifted) mass of the binary with component masses $m_{1} \geq m_{2}$, and we set $G=c=1$. Reference [32] shows that this improvement can be attributed to the waveform being more sensitive to variations in the orbital-plane spin angles near the merger. In particular, measuring the spins near the merger leads to improved constraints for $\phi_{1}$ and $\phi_{2}$ for several events in the latest GWTC-2 catalog [33-37] released by the LIGO-Virgo Collaboration. While the $\Delta \phi$ measurements are not significantly impacted, Ref. [32] shows that this parameter will also be better constrained with louder signals expected in the future.

In this work, we use the spin constraints from Ref. [32] to perform the first measurement of the full spin distribution in the astrophysical binary $\mathrm{BH}$ population. In particular, we identify a preference for $\Delta \phi \sim \pm \pi$, which can be a signature of SORs. Next, given the spin population, we derive constraints on the kick population. Finally, we use the kick constraints to estimate the fraction of merger remnants retained by globular and nuclear star clusters.

Methodology.-The first step in our analysis is to estimate the binary $\mathrm{BH}$ parameters from individual $\mathrm{GW}$ signals, which is done following Bayes' theorem [38]:

$$
p(\Theta \mid d) \propto \mathcal{L}(d \mid \Theta) \pi(\Theta),
$$

where $p(\Theta \mid d)$ is the "posterior" probability distribution of the binary parameters $\Theta$ given the observed data $d, \mathcal{L}(d \mid \Theta)$ is the "likelihood" of the data given $\Theta$, and $\pi(\Theta)$ is the "prior" probability distribution for $\Theta$. The full set of binary parameters $\Theta$ is 15 dimensional [33] and includes the masses and spins of the component BHs as well as extrinsic properties such as the distance and sky location.

In this work, we use the posteriors samples from Ref. [32], obtained using the numerical relativity (NR) surrogate waveform model NRSur7dq4 [4], with the spins measured at $t_{\text {ref }} / M=-100$. NRSur7dq4 accurately reproduces precessing NR simulations and is necessary to reliably measure the orbital-plane spin angles [32]. GWTC-2 includes a total of 46 binary BH events. However, because NRSur7dq4 only encompasses $\sim 20$ orbits before merger, it can only be applied to the shorter signals with $M \gtrsim 60 \mathrm{M}_{\odot}$ [4]. This reduces our set of events to 31; these events are listed in Table I of Ref. [32].

Given the posterior samples $p(\Theta \mid d)$ for the individual events, we want to measure the astrophysical distribution of the full spin degrees of freedom, $\mathbb{S}=\left\{\chi_{1}, \chi_{2}, \theta_{1}, \theta_{2}, \phi_{1}\right.$, $\Delta \phi\}$, which is a subset of $\Theta$. The remaining angle, $\phi_{2}$, is redundant given $\phi_{1}$ and $\Delta \phi$; we choose to work with $\Delta \phi$ as it is relevant for SORs. As an intermediate step, we first reweight the posterior samples for each event to account for known astrophysical constraints on the primary mass and mass ratio $\left(q=m_{2} / m_{1}\right)$ populations [12]. The details of the reweighting procedure are given in the Supplemental Material [39]. After reweighting, Eq. (1) can be rewritten as

$$
p(\Theta \mid d, R) \propto \mathcal{L}(d \mid \Theta) \pi(\Theta \mid R),
$$

where $R$ indicates that these are the reweighted posteriors. Using these reweighted posteriors in Eq. (4) below ensures that the implicit priors on the mass population are astrophysically motivated [50].

To constrain the astrophysical distribution of $\mathbb{S}$, we begin by making the assumption that the true value of $\mathbb{S}$ for each event is drawn from a common underlying distribution $\pi(\mathbb{S} \mid \Lambda)$, which is conditional on a set of "hyperparameters" $\Lambda$. We then use hierarchical Bayesian inference [38] to collectively analyze all 31 events and constrain $\Lambda$ :

$$
p\left(\Lambda \mid\left\{d_{i}\right\}\right) \propto \mathcal{L}\left(\left\{d_{i}\right\} \mid \Lambda\right) \pi(\Lambda),
$$

where $p\left(\Lambda \mid\left\{d_{i}\right\}\right)$ is the "hyperposterior" distribution for $\Lambda$ given a set of observations $\left\{d_{i}\right\}, \mathcal{L}\left(\left\{d_{i}\right\} \mid \Lambda\right)$ is the "hyperlikelihood" of this dataset given $\Lambda$, and $\pi(\Lambda)$ is the "hyperprior" distribution for $\Lambda$. In our case, $\left\{d_{i}\right\}$ with $i=$ $1 \ldots N$ represents the observed data for our set of $N=$ $31 \mathrm{GW}$ events. The hyperlikelihood is obtained by coherently combining the data from all events [38]:

$$
\mathcal{L}\left(\left\{d_{i}\right\} \mid \Lambda\right) \propto \prod_{i}^{N} \int d \mathbb{S}_{i} p\left(\mathbb{S}_{i} \mid d_{i}, R\right) \frac{\pi\left(\mathbb{S}_{i} \mid \Lambda\right)}{\pi\left(\mathbb{S}_{i} \mid R\right)} .
$$

For the underlying distribution $\pi(\mathbb{S} \mid \Lambda)$, the spin magnitudes and tilts are modeled following the "default spin" 
model of Ref. [12]. The orbital-plane spin angles $\phi_{1}$ and $\Delta \phi$ are modeled as being drawn from independent von Mises distributions [51]. The von Mises distribution is an approximation of a Gaussian distribution with periodic boundary conditions and is parameterized by a mean and a standard deviation (or simply, width).

The explicit forms of $\pi(\mathbb{S} \mid \Lambda)$ and the hyperprior $\pi(\Lambda)$ are given in the Supplemental Material [39]. In particular, the priors on the mean $\left(\mu_{\phi_{1}}\right.$ and $\left.\mu_{\Delta \phi}\right)$ and width $\left(\sigma_{\phi_{1}}\right.$ and $\left.\sigma_{\Delta \phi}\right)$ hyperparameters for the $\phi_{1}$ and $\Delta \phi$ distributions are as follows. The prior for the mean parameters is always uniform in $(-\pi, \pi)$. We consider two different prior choices for the widths: (i) a Jeffreys prior [52] that is log-uniform in $\sigma_{\phi_{1}}$ and $\sigma_{\Delta \phi}$ between $(0.3,4 \pi)$, henceforth referred to as the JEFFREYS- $\sigma_{\phi}$ prior; and (ii) a prior that is uniform in $\sigma_{\phi_{1}}$ and $\sigma_{\Delta \phi}$ between $(0.3,4 \pi)$, henceforth referred to as the FLAT$\sigma_{\phi}$ prior. The JEFFREYS prior is an uninformative prior choice often used for scale parameters [52]. The FLAT prior may be considered a control case to understand the impact of the prior. The lower limit of $0.3 \mathrm{rad}$ on the width priors is arbitrary but chosen to be smaller than the sharpest features we expect to be resolvable with LIGO-Virgo (which we estimate from the NR injections in Ref. [32]). The upper limit of $4 \pi$ is chosen to be large enough to approximate a flat distribution between $(-\pi, \pi)$.

We use the BILBY [53] package with the DYNESTY [54] sampler to draw posterior samples for the hyperparameters $\Lambda$ from $p\left(\Lambda \mid\left\{d_{i}\right\}\right)$. Finally, the posterior distribution for the
$\mathbb{S}$ population, also referred to as the "posterior population distribution," is obtained by averaging over $\Lambda$ [38]:

$$
p(\mathbb{S})=\int d \Lambda \pi(\mathbb{S} \mid \Lambda) p\left(\Lambda \mid\left\{d_{i}\right\}\right)
$$

In practice, this is done by drawing samples from the hyperposterior $p\left(\Lambda \mid\left\{d_{i}\right\}\right)$ and evaluating $\pi(\mathbb{S} \mid \Lambda)$ on an array of $\mathbb{S}$ values for each $\Lambda$ sample. This gives us an ensemble of probability distributions on $\mathbb{S}$, which we use to compute the mean and $90 \%$ credible widths. Finally, we note that we ignore selection effects for the spin population, as they are not expected to be significant at current sensitivity [39].

Spin population.-Figure 2 shows our constraints on the $\phi_{1}$ and $\Delta \phi$ populations. The left panel shows the posteriors for the mean and width hyperparameters. For both JEFFREYS- $\sigma_{\phi}$ and FLAT- $\sigma_{\phi}$ prior choices, we find that the 1D marginalized posteriors for the widths $\sigma_{\phi_{1}}$ and $\sigma_{\Delta \phi}$ are dominated by the prior itself. However, the $1 \mathrm{D}$ posteriors for the mean parameters show a preference for $\mu_{\phi_{1}} \sim-\pi / 4$ and $\mu_{\Delta \phi} \sim \pm \pi$. This is reflected in the corresponding constraints on the posterior population distributions, $p\left(\phi_{1}\right)$ and $p(\Delta \phi)$, shown in the right panel of Fig. 2. These represent our constraints on the astrophysical distributions for $\phi_{1}$ and $\Delta \phi$; they are generated by evaluating the von Mises model using draws from the joint posterior of the mean and width parameters.

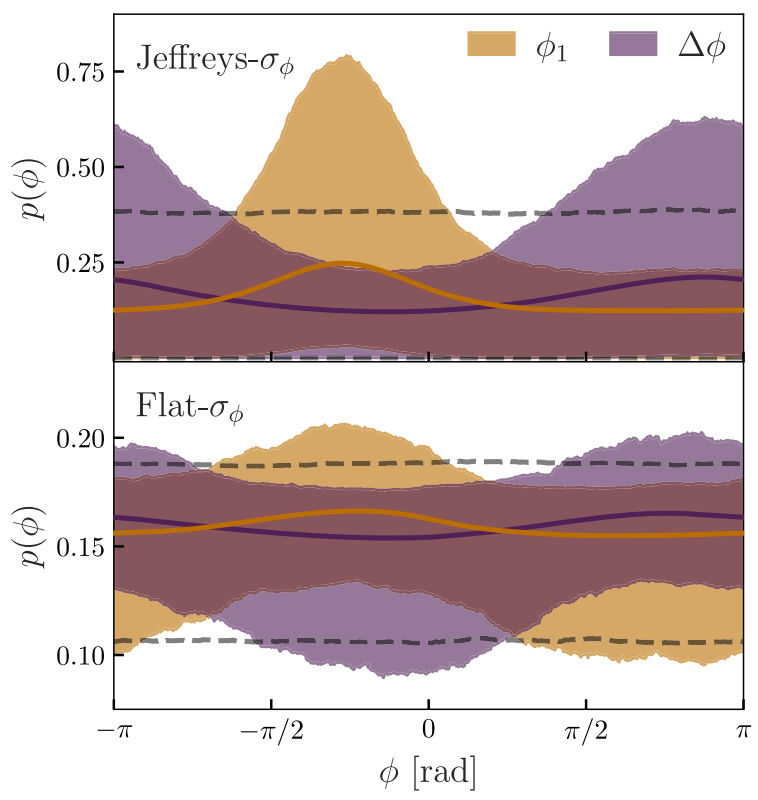

FIG. 2. Constraints on the $\phi_{1}$ and $\Delta \phi$ populations at $t_{\text {ref }} / M=-100$. Left: Posteriors for the mean and width parameters. The shaded regions show $90 \%$ credible bounds on joint 2D posteriors. The diagonal plots show $1 \mathrm{D}$ marginalized posteriors, with the priors shown as dashed black lines. We consider two prior choices (JEFFREYS- $\sigma_{\phi}$ and FLAT- $\sigma_{\phi}$ ) for the width parameters. Right: Constraints on the posterior population distributions $p\left(\phi_{1}\right)$ and $p(\Delta \phi)$ for the two prior choices. Shaded regions show $90 \%$ credible bounds, while the solid lines show the mean. The dashed gray lines show the $90 \%$ prior bounds. 
We interpret the population constraint in Fig. 2 as follows. For the FLAT $-\sigma_{\phi}$ prior, examining the 2D posterior for $\mu_{\Delta \phi}-\sigma_{\Delta \phi}$, we note that when $\sigma_{\Delta \phi} \rightarrow 0$, only the region around $\mu_{\Delta \phi} \sim \pm \pi$ is allowed in the $90 \%$ credible region. This means that, if there is a sharp peak in the $\Delta \phi$ population, it is only allowed near $\sim \pm \pi$. Similarly, examining the $2 \mathrm{D}$ posterior for $\mu_{\phi_{1}}-\sigma_{\phi_{1}}$, we find that when $\sigma_{\phi_{1}} \rightarrow 0$, there is a preference for $\mu_{\phi_{1}} \sim-\pi / 4$. The preferences in the 1D $\mu_{\phi_{1}} / \mu_{\Delta \phi}$ posteriors and the $p\left(\phi_{1}\right) / p(\Delta \phi)$ distributions get amplified for the JEFFREYS- $\sigma_{\phi}$ prior, as this prior already prefers small widths. In short, the data disfavor peaks at regions other than $\phi_{1} \sim-\pi / 4$ and $\Delta \phi \sim \pm \pi$, and this leads to $p\left(\phi_{1}\right)$ and $p(\Delta \phi)$ peaks in these regions. However, the data are not informative enough to constrain the widths of these peaks. We further note that both populations are still consistent with a uniform distribution at the $90 \%$ credible level.

It is important to recognize that the location of the $\phi_{1}$ peak in Fig. 2 depends strongly on our choice of reference point. This is because $\phi_{1}$ changes on the orbital timescale as it is defined with respect to the line of separation (cf. Fig. 1). On the other hand, $\Delta \phi$ only changes on the longer precession timescale, and we find that repeating our analysis using spins measured at $20 \mathrm{~Hz}$ leads to consistent results for the $\Delta \phi$ population [39]. However, the biggest gain in measuring the spins at $t_{\text {ref }} / M=-100$ is in the $\phi_{1}$ population constraint, as $\phi_{1}$ is significantly better measured there [32]. Constraining both $\phi_{1}$ and $\Delta \phi$ is necessary to constrain the kick population below.

For completeness, we include our constraints on the spin magnitude and tilt populations, along with full model hyperparameter posteriors in the Supplemental Material [39]. Our constraints on the spin magnitude and tilt populations are consistent with Ref. [12], and we do not find any obvious correlations between the orbital-plane spin angles and the other spin parameters. To gain further confidence in our results, we also conduct some mock population studies [39], which suggest that at least some $\phi_{1}$ and $\Delta \phi$ populations can be reliably recovered at current detector sensitivity. Finally, by iteratively leaving one event out from the dataset and repeating our analysis, we check that our results are not driven by any single event.

One limitation of this work is the restriction to the 31 signals with $M \gtrsim 60 \mathrm{M}_{\odot}$ so that we can use the NRSur7dq4 model. We also repeat our analysis for all 46 binary BH events from GWTC-2, using the phenomenological waveform model IMRPhenomTPHM [55] for the remaining 15 events. Interestingly, we find that there is some information gain in the width parameters in this case, with a preference for small widths. However, as noted in Ref. [32], IMRPhenomTPHM can have biases in recovering the orbitalplane spin angles. Therefore, while we include these results in the Supplemental Material [39] for completeness, we treat Fig. 2 as our main result.
Kick population.-Having constrained the full spin degrees of freedom for the binary BH population, we can now derive constraints on the kick population. We begin by generating one realization of the $q, \chi_{1}$, and $\chi_{2}$ populations. For $q$, we use the same model that was used in the initial posterior reweighting. For $\chi_{1,2}$, we use the spin population constraints at $t_{\text {ref }} / M=-100$. We simply draw one hyperparameter sample from the posterior of the hierarchical analysis and evaluate the $q, \chi_{1}$, and $\chi_{2}$ population models at that point. Next, we draw a large number of samples for $q$, $\chi_{1}$, and $\chi_{2}$ from this population realization and compute the corresponding kick magnitudes using the NRSur7dq4Remnant model $[4,56]$. Repeating these steps over many draws of $q$, $\chi_{1}$, and $\chi_{2}$ populations, we generate an ensemble of kick population distributions $p\left(v_{f}\right)$. For comparison, we also evaluate the prior $p\left(v_{f}\right)$ by repeating this procedure using prior hyperparameter samples.

Figure 3 shows the $90 \%$ credible constraints on $p\left(v_{f}\right)$ for the JEFFREYS- $\sigma_{\phi}$ and FLAT- $\sigma_{\phi}$ prior choices. In addition, we consider a prior choice where the $\phi_{1}$ and $\Delta \phi$ populations are restricted to be uniformly distributed. We refer to this prior choice as INFINITE- $\sigma_{\phi}$, as the other priors reduce to this when $\sigma_{\phi_{1}}=\sigma_{\Delta \phi}=\infty$. This restricted prior was also used in Ref. [57] to constrain the kick population. The mass ratio, spin magnitude, and tilt population models are the same for all three choices. We compare our kick population constraints against fiducial escape velocities for globular clusters [58,59], nuclear star clusters [59], elliptical galaxies [22], and Milky Way-like galaxies [60].

Comparing the prior and posterior ranges in Fig. 3, we note that there is significant information gain about the kick population, even though individual events are largely

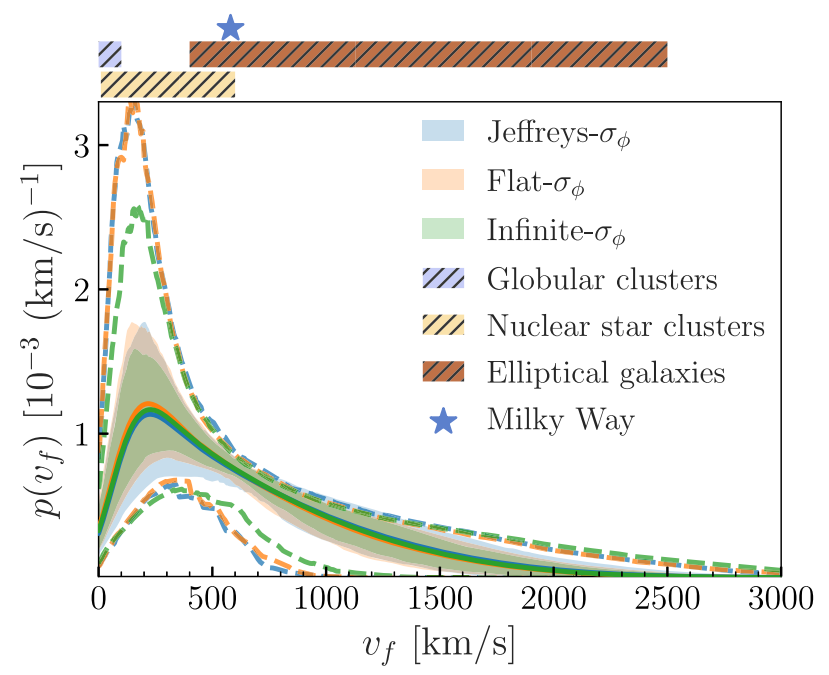

FIG. 3. Constraints on the kick magnitude population for different prior choices for $\sigma_{\phi_{1}}$ and $\sigma_{\Delta \phi}$. Shaded regions show the central $90 \%$ credible bounds for the posterior, while the solid lines show the mean. The dashed lines show the $90 \%$ bounds for the prior. For comparison, we show known ranges for the escape velocities for various types of host environments. 
uninformative about the kick [23]. The three prior choices lead to consistent kick populations in Fig. 3, with the INFINITE- $\sigma_{\phi}$ prior leading to the tightest constraint. This is expected as the INFINITE- $\sigma_{\phi}$ prior is a special case of the other two. This is also reflected in the more restrictive $p\left(v_{f}\right)$ prior in Fig. 3 for INFINITE- $\sigma_{\phi}$. It is somewhat surprising that the kick population is not hugely influenced by the prior choices on $\phi_{1}$ and $\Delta \phi$ population, even though the kick is known to be very sensitive to these parameters [25]. This is explained by the fact that the $\phi_{1}$ and $\Delta \phi$ distributions in Fig. 2 are still consistent with a uniform distribution at $90 \%$ credibility. We expect this to change with future observations.

Astrophysical implications. - While the location of the $\phi_{1}$ peak in Fig. 2 is not particularly important, the fact that there is a peak at all is indeed interesting. This feature has not been predicted for any formation channel. Therefore, our naive expectation is that this peak will get smoothed over as more data are added. However, it will be interesting to see if there are alternative explanations.

On the other hand, the preference for $\Delta \phi \sim \pm \pi$ in Fig. 2 is expected in some formation channels where SORs [6] are important. In particular, stellar binaries with significant supernova natal kicks and efficient stellar tides can be driven toward these resonances [13,14]. In the standard scenario where the heavier star becomes the heavier $\mathrm{BH}$, the $\Delta \phi \sim \pm \pi$ resonant mode is expected to be dominant. However, if mass transfer between the two components is significant, a mass-ratio reversal occurs and the $\Delta \phi \sim 0$ mode becomes dominant [13]. Note that the predictions of Refs. [13,14] are at $f_{\text {ref }}=20 \mathrm{~Hz}$, while our best constraints are at $t_{\text {ref }} / M=-100$. However, as $\Delta \phi$ only evolves on the precession timescale, we expect that a preference for $\Delta \phi \sim$ 0 or $\pm \pi$ at $f_{\text {ref }}=20 \mathrm{~Hz}$ leads to a similar preference at $t_{\text {ref }} / M=-100$. It will be interesting to extend the analysis of Refs. [13,14] to $t_{\text {ref }} / M=-100$, for example, using the spin dynamics of NRSur7dq4 [4].

While our $\Delta \phi$ population constraint can be interpreted as coming from SORs, this does not yet constitute conclusive evidence for them-especially not without a measurement of the width of the distribution to confirm this feature. In addition, consider a binary for which the spin angular momenta in the plane of the orbit perfectly cancel (which requires $\Delta \phi= \pm \pi$ ). This system will not undergo orbital precession and can approximately mimic a spinaligned system, as pointed out by Ref. [31]. As a result, a precessing waveform model can sometimes mistake a spinaligned system for one with $\Delta \phi= \pm \pi$. However, such a binary still undergoes spin precession that can be used to break this degeneracy given a sufficient signal-to-noise ratio. A more detailed analysis may be necessary to account for effects of such potential degeneracies on our results.

Finally, as an application of our $p\left(v_{f}\right)$ constraints in Fig. 3, we estimate the fraction of merger remnants that would be retained by various host environments. Assuming a maximum escape velocity $v_{\mathrm{esc}}^{\max }=100 \mathrm{~km} / \mathrm{s}$ for globular clusters $[58,59], 6_{-3}^{+5} \%\left(6_{-3}^{+4} \%\right)$ of the remnants will be retained for the JEFFREYS- $\sigma_{\phi}$ (FLAT- $\sigma_{\phi}$ ) prior. For nuclear star clusters, assuming $v_{\mathrm{esc}}^{\max }=600 \mathrm{~km} / \mathrm{s}$ [59], the retention fraction is constrained to $54_{-17}^{+19} \%\left(57_{-15}^{+16} \%\right)$ for the JEFFREYS- $\sigma_{\phi}\left(\right.$ FLAT- $\left.\sigma_{\phi}\right)$ prior. Averaging over the two prior choices, we estimate the retention fraction to be $\sim 3 \%-$ $10 \%$ for globular clusters, and $\sim 39 \%-73 \%$ for nuclear star clusters. All constraints are quoted at $90 \%$ credibility. Our constraints on the retention fraction are consistent with those of Refs. [57,61].

If the observed $\Delta \phi \sim \pm \pi$ preference is confirmed to be due to SORs, our findings have several important implications: (i) SOR measurements can be used to place new astrophysical constraints on supernova natal kicks and stellar tides [13,14]; (ii) SORs can be used to constrain the cosmic merger rate of isolated stellar binaries in galactic fields, as well as measure what fraction of merging binaries form via that channel; and (iii) the $\Delta \phi \sim \pm \pi$ resonance mode tends to enhance merger kicks and suppress the spins of the remnant BHs $[62,63]$, both of which are important observables for constraining the formation of heavy BHs via successive mergers [24]. Furthermore, independent of whether our findings can be attributed to SORs, our $p\left(v_{f}\right)$ constraints already suggest that globular clusters are an unlikely site for that formation channel.

Conclusion.-We constrain the distribution of the orbital-plane spin orientations $\phi_{1}$ and $\Delta \phi$ in the binary black hole population. We find that there is a preference for $\Delta \phi \sim \pm \pi$, which can be a signature of SORs. In addition, we find a preference for $\phi_{1} \sim-\pi / 4$ in the population, which has not been predicted for any astrophysical formation channel. However, the strength of these preferences depends on our prior choices. Finally, we constrain the distribution of recoil kicks in the population and estimate the fraction of merger remnants retained by globular and nuclear star clusters. We make our population constraints publicly available at Ref. [64].

Observational evidence for SORs has far reaching implications for black hole astrophysics. While our population constraints suggest the influence of SORs, we are unable to constrain the widths of the $\phi_{1}$ and $\Delta \phi$ distributions with the current dataset of events. Therefore, further observations are necessary to confirm these trends. With LIGO and Virgo approaching their design sensitivities [65], our constraints are certain to improve in the near future.

We thank Davide Gerosa and Katerina Chatziioannou for useful discussions. V. V. was supported by a Klarman Fellowship at Cornell. This project has received funding from the European Union's Horizon 2020 research and innovation program under the Marie Skłodowska-Curie Grant agreement No. 896869. S. B., M. I., and S. V. acknowledge the support of the National Science Foundation and the LIGO Laboratory. S. B. is also 
supported by the NSF Graduate Research Fellowship under Grant No. DGE-1122374. M. I. is supported by NASA through the NASA Hubble Fellowship Grant No. HSTHF2-51410.001-A awarded by the Space Telescope Science Institute, which is operated by the Association of Universities for Research in Astronomy, Inc., for NASA, under Contract NAS5-26555. This research made use of data, software, and/or web tools obtained from the Gravitational Wave Open Science Center [66], a service of the LIGO Laboratory, the LIGO Scientific Collaboration, and the Virgo Collaboration. LIGO was constructed by the California Institute of Technology and Massachusetts Institute of Technology with funding from the National Science Foundation and operates under Cooperative Agreement No. PHY-1764464. Computations were performed on the Alice cluster at ICTS; the Nemo cluster at University of Wisconsin-Milwaukee, which is supported by NSF Grant PHY-1626190; the Wheeler cluster at Caltech, which is supported by the Sherman Fairchild Foundation and by Caltech; and the High Performance Cluster at Caltech.

*Corresponding author. vijay.varma@aei.mpg.de

[1] T. A. Apostolatos, C. Cutler, G. J. Sussman, and K. S. Thorne, Spin-induced orbital precession and its modulation of the gravitational waveforms from merging binaries, Phys. Rev. D 49, 6274 (1994).

[2] L. E. Kidder, Coalescing binary systems of compact objects to postNewtonian 5/2 order. 5. Spin effects, Phys. Rev. D 52, 821 (1995).

[3] More precisely, we use the co-orbital frame defined in Ref. [4]. In this frame, the $z$ axis is along the direction that maximizes the power in the $(2,2)$ mode, which is taken to be the direction of the orbital angular momentum [5]. The $x$ axis is along the line of separation from the lighter to the heavier $\mathrm{BH}$, and the $y$ axis completes the right-handed triad. Note that this frame is defined using the gaugeinvariant waveform at future null infinity, rather than the gauge-dependent $\mathrm{BH}$ trajectories.

[4] V. Varma, S. E. Field, M. A. Scheel, J. Blackman, D. Gerosa, L. C. Stein, L. E. Kidder, and H. P. Pfeiffer, Surrogate models for precessing binary black hole simulations with unequal masses, Phys. Rev. Research 1, 033015 (2019).

[5] M. Boyle, R. Owen, and H. P. Pfeiffer, A geometric approach to the precession of compact binaries, Phys. Rev. D 84, 124011 (2011).

[6] J. D. Schnittman, Spin-orbit resonance and the evolution of compact binary systems, Phys. Rev. D 70, 124020 (2004).

[7] M. Kesden, D. Gerosa, R. O’Shaughnessy, E. Berti, and U. Sperhake, Effective Potentials and Morphological Transitions for Binary Black-Hole Spin Precession, Phys. Rev. Lett. 114, 081103 (2015).

[8] D. Gerosa, M. Kesden, U. Sperhake, E. Berti, and R. O'Shaughnessy, Multi-timescale analysis of phase transitions in precessing black-hole binaries, Phys. Rev. D 92, 064016 (2015).
[9] D. Gerosa, R. O’Shaughnessy, M. Kesden, E. Berti, and U. Sperhake, Distinguishing black-hole spin-orbit resonances by their gravitational-wave signatures, Phys. Rev. D 89, 124025 (2014).

[10] D. Trifirò, R. O'Shaughnessy, D. Gerosa, E. Berti, M. Kesden, T. Littenberg, and U. Sperhake, Distinguishing black-hole spin-orbit resonances by their gravitational wave signatures. II: Full parameter estimation, Phys. Rev. D 93, 044071 (2016).

[11] C. Afle, A. Gupta, B. Gadre, P. Kumar, N. Demos, G. Lovelace, H. G. Choi, H. M. Lee, S. Mitra, M. Boyle, D. A. Hemberger, L. E. Kidder, H. P. Pfeiffer, M. A. Scheel, and B. Szilagyi, Detection and characterization of spin-orbit resonances in the advanced gravitational wave detectors era, Phys. Rev. D 98, 083014 (2018).

[12] R. Abbott et al. (LIGO Scientific, Virgo Collaborations), Population properties of compact objects from the Second LIGO-Virgo gravitational-wave transient catalog, Astrophys. J. Lett. 913, L7 (2021).

[13] D. Gerosa, M. Kesden, E. Berti, R. O'Shaughnessy, and U. Sperhake, Resonant-plane locking and spin alignment in stellar-mass black-hole binaries: A diagnostic of compactbinary formation, Phys. Rev. D 87, 104028 (2013).

[14] D. Gerosa, E. Berti, R. O'Shaughnessy, K. Belczynski, M. Kesden, D. Wysocki, and W. Gladysz, Spin orientations of merging black holes formed from the evolution of stellar binaries, Phys. Rev. D 98, 084036 (2018).

[15] W. B. Bonnor, M. A. Rotenberg, and R. Louis, Transport of momentum by gravitational waves: The linear approximation, Proc. R. Soc. A. 265, 109 (1961).

[16] A. Peres, Classical radiation recoil, Phys. Rev. 128, 2471 (1962).

[17] J. D. Bekenstein, Gravitational-radiation recoil and runaway black holes, Astrophys. J. 183, 657 (1973).

[18] M. J. Fitchett, The influence of gravitational wave momentum losses on the centre of mass motion of a Newtonian binary system, Mon. Not. R. Astron. Soc. 203, 1049 (1983).

[19] M. Campanelli, C. O. Lousto, Y. Zlochower, and D. Merritt, Maximum Gravitational Recoil, Phys. Rev. Lett. 98, 231102 (2007).

[20] J. A. Gonzalez, M. Hannam, U. Sperhake, B. Brugmann, and S. Husa, Supermassive Recoil Velocities for Binary Black-Hole Mergers with Antialigned Spins, Phys. Rev. Lett. 98, 231101 (2007).

[21] C. O. Lousto and Y. Zlochower, Hangup Kicks: Still Larger Recoils by Partial Spin/Orbit Alignment of Black-Hole Binaries, Phys. Rev. Lett. 107, 231102 (2011).

[22] D. Merritt, M. Milosavljevic, M. Favata, S. A. Hughes, and D. E. Holz, Consequences of gravitational radiation recoil, Astrophys. J. 607, L9 (2004).

[23] V. Varma, M. Isi, and S. Biscoveanu, Extracting the Gravitational Recoil from Black Hole Merger Signals, Phys. Rev. Lett. 124, 101104 (2020).

[24] D. Gerosa and M. Fishbach, Hierarchical mergers of stellarmass black holes and their gravitational-wave signatures, Nat. Astron. 5, 749 (2021).

[25] B. Brugmann, J. A. Gonzalez, M. Hannam, S. Husa, and U. Sperhake, Exploring black hole superkicks, Phys. Rev. D 77, 124047 (2008). 
[26] J. Aasi et al. (LIGO Scientific Collaboration), Advanced LIGO, Classical Quantum Gravity 32, 074001 (2015).

[27] F. Acernese et al. (Virgo Collaboration), Advanced Virgo: A second-generation interferometric gravitational wave detector, Classical Quantum Gravity 32, 024001 (2015).

[28] B. P. Abbott et al. (LIGO Scientific, Virgo Collaborations), Binary black hole population properties inferred from the first and second observing runs of Advanced LIGO and Advanced Virgo, Astrophys. J. Lett. 882, L24 (2019).

[29] S. Vitale, R. Lynch, J. Veitch, V. Raymond, and R. Sturani, Measuring the Spin of Black Holes in Binary Systems using Gravitational Waves, Phys. Rev. Lett. 112, 251101 (2014).

[30] P. Schmidt, F. Ohme, and M. Hannam, Towards models of gravitational waveforms from generic binaries II: Modelling precession effects with a single effective precession parameter, Phys. Rev. D 91, 024043 (2015).

[31] S. Biscoveanu, M. Isi, V. Varma, and S. Vitale, Measuring the spins of heavy binary black holes, Phys. Rev. D 104, 103018 (2021).

[32] V. Varma, M. Isi, S. Biscoveanu, W. M. Farr, and S. Vitale, companion paper, Measuring binary black hole orbitalplane spin orientations, Phys. Rev. D 105, 024045 (2022).

[33] R. Abbott et al. (LIGO Scientific, Virgo Collaborations), GWTC-2: Compact Binary Coalescences Observed by LIGO and Virgo during the First Half of the Third Observing Run, Phys. Rev. X 11, 021053 (2021).

[34] B. P. Abbott et al. (LIGO Scientific, Virgo Collaborations), GWTC-1: A Gravitational-Wave Transient Catalog of Compact Binary Mergers Observed by LIGO and Virgo during the First and Second Observing Runs, Phys. Rev. X 9, 031040 (2019).

[35] R. Abbott et al. (LIGO Scientific, Virgo Collaborations), Open data from the first and second observing runs of Advanced LIGO and Advanced Virgo, SoftwareX 13, 100658 (2021).

[36] LIGO Scientific and Virgo Collaborations, GWTC-1, 10.7935/82H3-HH23 (2018).

[37] LIGO Scientific and Virgo Collaborations, GWTC-2, 10.7935/99gf-ax93 (2020).

[38] E. Thrane and C. Talbot, An introduction to Bayesian inference in gravitational-wave astronomy: Parameter estimation, model selection, and hierarchical models, Pub. Astron. Soc. Aust. 36, e010 (2019).

[39] See Supplemental Material, which includes Refs. [40-49], at http://link.aps.org/supplemental/10.1103/PhysRevLett .128 .031101 for details about our hierarchical analysis, posterior for the full spin population, population constraints at $20 \mathrm{~Hz}$, a mock population study, and results for all GWTC-2 events (using a phenomenological model when the surrogate is not applicable).

[40] S. Galaudage, C. Talbot, and E. Thrane, Gravitational-wave inference in the catalog era: Evolving priors and marginal events, Phys. Rev. D 102, 083026 (2020).

[41] I. M. Romero-Shaw et al., Bayesian inference for compact binary coalescences with bilby: Validation and application to the first LIGO-Virgo gravitational-wave transient catalogue, Mon. Not. R. Astron. Soc. 499, 3295 (2020).

[42] D. Wysocki, J. Lange, and R. O’Shaughnessy, Reconstructing phenomenological distributions of compact binaries via gravitational wave observations, Phys. Rev. D 100, 043012 (2019).

[43] C. Talbot and E. Thrane, Determining the population properties of spinning black holes, Phys. Rev. D 96, 023012 (2017).

[44] R. Abbott et al. (LIGO Scientific, Virgo Collaborations), GW190521: A Binary Black Hole Merger with a Total Mass of $150 M_{\odot}$, Phys. Rev. Lett. 125, 101102 (2020).

[45] J. Veitch, V. Raymond, B. Farr, W. Farr, P. Graff et al., Robust parameter estimation for compact binaries with ground-based gravitational-wave observations using the LALInference software library, Phys. Rev. D 91, 042003 (2015).

[46] V. Varma, S. E. Field, M. A. Scheel, J. Blackman, L. E. Kidder, and H. P. Pfeiffer, Surrogate model of hybridized numerical relativity binary black hole waveforms, Phys. Rev. D 99, 064045 (2019).

[47] K. Alvi, Energy and angular momentum flow into a black hole in a binary, Phys. Rev. D 64, 104020 (2001).

[48] E. Poisson, Absorption of mass and angular momentum by a black hole: Time-domain formalisms for gravitational perturbations, and the small-hole / slow-motion approximation, Phys. Rev. D 70, 084044 (2004).

[49] J. Blackman, S. E. Field, M. A. Scheel, C. R. Galley, C. D. Ott, M. Boyle, L. E. Kidder, H. P. Pfeiffer, and Béla Szilágyi, Numerical relativity waveform surrogate model for generically precessing binary black hole mergers, Phys. Rev. D 96, 024058 (2017).

[50] S. Vitale, D. Gerosa, W. M. Farr, and S. R. Taylor, Inferring the properties of a population of compact binaries in presence of selection effects, arXiv:2007.05579.

[51] K. V. Mardia and P. E. Jupp, Directional Statistics (John Wiley and Sons, Ltd., West Sussex, 1999).

[52] H. Jeffreys, An invariant form for the prior probability in estimation problems, Proc. R. Soc. A 186, 453 (1946).

[53] G. Ashton et al., BILBY: A user-friendly Bayesian inference library for gravitational-wave astronomy, Astrophys. J. Suppl. Ser. 241, 27 (2019).

[54] J. S. Speagle, DYNESTY: A dynamic nested sampling package for estimating Bayesian posteriors and evidences, Mon. Not. R. Astron. Soc. 493, 3132 (2020).

[55] H. Estellés, M. Colleoni, C. García-Quirós, S. Husa, D. Keitel, M. Mateu-Lucena, M. de Lluc Planas, and A. Ramos-Buades, New twists in compact binary waveform modelling: A fast time domain model for precession, arXiv:2105.05872.

[56] V. Varma, D. Gerosa, L. C. Stein, F. Hébert, and H. Zhang, High-Accuracy Mass, Spin, and Recoil Predictions of Generic Black-Hole Merger Remnants, Phys. Rev. Lett. 122, 011101 (2019).

[57] Z. Doctor, B. Farr, and D. E. Holz, Black hole leftovers: The remnant population from binary black hole mergers, Astrophys. J. Lett. 914, L18 (2021).

[58] O. Y. Gnedin, H. Zhao, J. E. Pringle, S. Michael Fall, M. Livio, and G. Meylan, The unique history of the globular cluster omega centauri, Astrophys. J. Lett. 568, L23 (2002).

[59] F. Antonini and Frederic A. Rasio, Merging black hole binaries in galactic nuclei: Implications for Advanced-LIGO detections, Astrophys. J. 831, 187 (2016). 
[60] G. Monari, B. Famaey, I. Carrillo, T. Piffl, M. Steinmetz, R. F. G. Wyse, F. Anders, C. Chiappini, and K. Janßen, The escape speed curve of the Galaxy obtained from Gaia DR2 implies a heavy Milky Way, Astron. Astrophys. 616, L9 (2018).

[61] P. Mahapatra, A. Gupta, M. Favata, K. G. Arun, and B. S. Sathyaprakash, Remnant black hole kicks and implications for hierarchical mergers, Astrophys. J. Lett. 918, L31 (2021).

[62] E. Berti, M. Kesden, and U. Sperhake, Effects of postNewtonian spin alignment on the distribution of black-hole recoils, Phys. Rev. D 85, 124049 (2012).
[63] M. Kesden, U. Sperhake, and E. Berti, Relativistic suppression of black hole recoils, Astrophys. J. 715, 1006 (2010).

[64] V. Varma, S. Biscoveanu, M. Isi, W. M. Farr, and S. Vitale, Public data release for hints of spin-orbit resonances in the binary black hole population, github.com/vijayvarma392/ spin_kick_pop_GWTC2.

[65] B.P. Abbott et al. (KAGRA, LIGO Scientific, VIRGO Collaborations), Prospects for observing and localizing gravitational-wave transients with Advanced LIGO, Advanced Virgo and KAGRA, Living Rev. Relativity 21, 3 (2018).

[66] LIGO Scientific and Virgo Collaborations, Gravitational wave open science center, https://www.gw-openscience.org. 